\title{
The Extended Cone $b$-Metric-like Spaces over Banach Algebra and Some Applications
}

\author{
Jerolina Fernandez ${ }^{1}$, Neeraj Malviya ${ }^{2}$, Ana Savić ${ }^{3}$, Marija Paunović ${ }^{4, *(D)}$ and Zoran D. Mitrović ${ }^{5}$ (i) \\ 1 Department of Science, The Bhopal School of Social Sciences, Bhopal 462024, India; \\ jerolinafernandez@bsssbhopal.edu.in \\ 2 Department of Mathematics, Government College, Timarni 461228, India; maths.neeraj@gmail.com \\ 3 School of Electrical and Computer Engineering of Applied Studies, Academy of Technical and Art Applied \\ Studies, 11070 Belgrade, Serbia; ana.savic@viser.edu.rs \\ 4 Faculty of Hotel Management and Tourism, University of Kragujevac, 36210 Kragujevac, Serbia \\ 5 Faculty of Electrical Engineering, University of Banja Luka, Patre 5, \\ 78000 Banja Luka, Bosnia and Herzegovina; zoran.mitrovic@etf.unibl.org \\ * Correspondence: majap@rcub.bg.ac.rs
}

Citation: Fernandez, J.; Malviya, N.; Savić, A.; Paunović, M.; Mitrović,

Z.D. The Extended Cone

$b$-Metric-like Spaces over Banach Algebra and Some Applications. Mathematics 2022, 10, 149. https:// doi.org/10.3390/math10010149

Academic Editors: Jin-Ting Zhang and Christopher Goodrich

Received: 26 November 2021

Accepted: 1 January 2022

Published: 4 January 2022

Publisher's Note: MDPI stays neutral with regard to jurisdictional claims in published maps and institutional affiliations.

Copyright: (C) 2022 by the authors Licensee MDPI, Basel, Switzerland. This article is an open access article distributed under the terms and conditions of the Creative Commons Attribution (CC BY) license (https:// creativecommons.org/licenses/by/ $4.0 /)$.

\begin{abstract}
In this paper, we introduce the structure of extended cone $b$-metric-like spaces over Banach algebra as a generalization of cone $b$-metric-like spaces over Banach algebra. In this generalized space we define the notion of generalized Lipschitz mappings in the setup of extended cone $b$-metric-like spaces over Banach algebra and investigated some fixed point results. We also provide examples to illustrate the results presented herein. Finally, as an application of our main result, we examine the existence and uniqueness of solution for a Fredholm integral equation.
\end{abstract}

Keywords: extended cone $b$-metric-like spaces over Banach algebra; generalized Lipschitz mapping; fixed point

\section{Introduction}

Fixed point theory is a well furnished concept and plays a fundamental role in analysis and topology. It has wide applications in different domains of mathematics such as in the theory of ODEs, PDEs, integral Equations and so forth. Metric space was introduced by Fréchet [1] and in the last 50 years it has become a dynamic area of research. There are various generalizations of metric space such as b-metric space, 2-metric space, G-metric space, fuzzy metric space, cone metric space, and so forth.

Huang and Zhang [2] generalized the concept of metric space and introduced cone metric space. They replaced the set of real numbers to real Banach space. Recently, many articles have discussed the results on cone metric spaces being identical to results on ordinary metric spaces.

Finally, Liu and Xu [3] introduced the concept of cone metric spaces over Banach algebras and proved Banach contraction principle in the setting of cone metric spaces over Banach algebras. The authors presented some fixed point theorems of generalized Lipschitz mappings in the new setting without the assumption of normality, which are not equivalent to metric spaces in terms of the existence of the fixed points of the mappings. Motivated and inspired by the research works mentioned above, in 2017, Fernandez et al. [4] proposed cone b-metric-like spaces over Banach algebra, a generalization of $b$-metric-like spaces and investigated the fixed point of generalized contractions and expansive mapping in the setting of cone $b$-metric-like spaces over Banach algebras with a nonnormal cone. More about the results on conus metric spaces and generalizations in those spaces can be seen in Fernandez et al. [5,6], Mitrović et al. [7-9], Roy et al. [10], Shatanawi et al. [11], Radenović and Rhoades [12], and Rezapour and Hamlbarani [13].

The purpose of this paper is to present the notion of an extended cone $b$-metric-like space over Banach algebra and investigate the existence of a fixed point for generalized 
Lipschitz maps. As an application, we study the existence and uniqueness of solution for a Fredholm integral equation. Our results generalize and improve the results in [4].

\section{Preliminaries}

Let $A$ always be a real Banach algebra such that

1. $(\varsigma \varrho) v=\varsigma(\varrho v)$,

2. $\varsigma(\varrho+v)=\varsigma \varrho+\varsigma v$ and $(\varsigma+\varrho) v=\varsigma v+\varrho v$,

3. $\alpha(\varsigma \varrho)=(\alpha \varsigma) \varrho=\varsigma(\alpha \varrho)$,

4. $\|\varsigma \varrho\| \leq\|\varsigma\|\|\varrho\|$,

for all $\varsigma, \varrho, v \in A, \alpha \in \mathbb{R}$.

If $e \varsigma=\varsigma e=\varsigma$ for all $\varsigma \in A$ then $e \in A$ is called unit (i.e., a multiplicative identity). If there is an element $\varsigma \in A$ such that $\zeta \varrho=\varrho \varsigma=e$, then $\varsigma \in A$ is said to be invertible. $\varsigma^{-1}$ is the inverse of $\varsigma$. For more details, we refer the readers to [14].

The following proposition is given in [14].

Proposition 1. Suppose that the spectral radius $\widehat{\rho}(\varsigma)$ of an element $\varsigma \in A$ is less than 1 , that is,

$$
\widehat{\rho}(\varsigma)=\lim _{n \rightarrow+\infty}\left\|\varsigma^{n}\right\|^{\frac{1}{n}}=\inf _{n \geq 1}\left\|\zeta^{n}\right\|^{\frac{1}{n}}<1,
$$

then $e-\varsigma$ is invertible, where $e \in A$ is a unit. Moreover, then $(e-\varsigma)$ is invertible. Actually,

$$
(e-\varsigma)^{-1}=\sum_{i=0}^{+\infty} \varsigma^{i} .
$$

Remark 1. From [14] we see that the spectral radius $\rho(\varsigma)$ of $\varsigma$ satisfies $\rho(\varsigma) \leq\|\varsigma\|$ for all $\varsigma \in A$, where $A$ is a Banach algebra with a unit e.

Remark 2. In Proposition 1, if the condition $\rho(\varsigma)<1$ is replaced by $\|\varsigma\| \leq 1$, then the conclusion remains true, ref. [15].

Remark 3. If $\rho(\varsigma)<1$ then $\left\|\varsigma^{n}\right\| \rightarrow 0 \quad(n \rightarrow+\infty)$ (see [15]).

Definition 1. A subset $P$ of $A$ is called a cone if:

1. $P$ is non-empty, closed and $\{\theta, e\} \subset P$;

2. $a \alpha+b \beta \subset P$ for all non-negative real numbers $\alpha, \beta$ and $a, b \geq 0$;

3. $P^{2}=P P \subset P$;

4. $P \cap(-P)=\{\theta\}$;

where $\theta$ denotes the null of the Banach algebra $A$.

For a given cone $P \subset A$, we can define a partial ordering $\preceq$ with respect to $P$ by $\varsigma \preceq \varrho$ if and only if $\varrho-\varsigma \in P, \varsigma \prec \varrho$ will stand for $\varsigma \preceq \varrho$ and $\varsigma \neq \varrho$, while $\varsigma \ll \varrho$ will stand for $\varrho-\varsigma \in$ int $P$, where int $P$ denotes the interior of $P$. If int $P \neq \varnothing$, then $P$ is called a solid cone. The cone $P$ is called normal if there is a number $\lambda>0$ such that, for all $\varsigma, \varrho \in A$,

$$
\theta \preceq \varsigma \preceq \varrho \Rightarrow\|\varsigma\| \leq \lambda\|\varrho\| .
$$

The least positive number satisfying the above is called the normal constant of $P$, ref. [2].

Definition 2. A cone b-metric on a nonempty set $M$ is a function $\sigma_{b}: M \times M \rightarrow \mathbb{R}^{+}$such that for all $\varsigma, Q, v \in M$ and a constant $s \geq 1$ the following conditions hold:

$\left(\sigma_{b_{1}}\right) \sigma_{b}(\varsigma, \varrho)=\theta \Leftrightarrow \varsigma=\varrho$,

$\left(\sigma_{b_{2}}\right) \sigma_{b}(\varsigma, \varrho)=\sigma_{b}(\varrho, \varsigma)$, 
$\left(\sigma_{b_{3}}\right) \sigma_{b}(\varsigma, \varrho) \preceq s\left[\sigma_{b}(\varsigma, v)+\sigma_{b}(v, \varrho)\right]$.

The pair $\left(M, \sigma_{b}\right)$ is then called a cone b-metric space.

Definition 3 (Ref. [4]). A cone b-metric-like on a nonempty set $M$ is a function $\sigma_{b}: M \times M \rightarrow A$ such that for all $\varsigma, Q, v \in M$ and $a$ constant $s \geq 1$ the following conditions hold:

$\left(\sigma_{b_{1}}\right) \sigma_{b}(\varsigma, \varrho)=\theta \Rightarrow \varsigma=\varrho$,

$\left(\sigma_{b_{2}}\right) \sigma_{b}(\varsigma, \varrho)=\sigma_{b}(\varrho, \varsigma)$,

$\left(\sigma_{b_{3}}\right) \quad \sigma_{b}(\varsigma, \varrho) \preceq s\left[\sigma_{b}(\varsigma, v)+\sigma_{b}(v, \varrho)\right]$.

The pair $\left(M, \sigma_{b}\right)$ is then called a cone b-metric-like space over Banach algebra A.

A cone $b$-metric-like on $M$ satisfies all of the conditions of a cone $b$-metric space except that $\sigma_{b}(\varsigma, \varsigma)$ need not be $\theta$ for $\varsigma \in M$.

\section{Extended Cone $\boldsymbol{b}$-Metric-Like Space over Banach Algebra}

We present a generalized cone $b$-metric-like space over Banach algebra namely an extended cone $b$-metric-like space over Banach algebra, as follows.

Definition 4. Let $M$ be a non-empty set, $\eta: M \times M \rightarrow[1,+\infty)$ be a mapping. A mapping $\sigma_{b}: M \times M \rightarrow A$ is said to be extended cone b-metric-like space over Banach algebra such that:

$\left(\sigma_{b} 1\right): \quad \sigma_{b}(\varsigma, \varrho)=\theta \Rightarrow \varsigma=\varrho$,

$\left(\sigma_{b} 2\right): \sigma_{b}(\varsigma, \varrho)=\sigma_{b}(\varrho, \varsigma)$

$\left(\sigma_{b} 3\right): \quad \sigma_{b}(\varsigma, \varrho) \preceq \eta(\varsigma, \varrho)\left[\sigma_{b}(\varsigma, v)+\sigma_{b}(v, \varrho)\right]$.

The pair $\left(M, \sigma_{b}\right)$ is called an extended cone $b$-metric-like space over Banach algebra.

Remark 4. An extended cone b-metric-like space over Banach algebra generalizes several known cone metric structures, such as:

(i) If $\eta(\varsigma, \varrho)=1$, for all $\varsigma, \varrho \in M$, then an extended cone $b$-metric-like space over Banach algebra reduces to a cone b-metric-like space over Banach algebra;

(ii) A cone b-metric-like space over Banach algebra is an extended cone b-metric-like space over Banach algebra for $\eta(\varsigma, \varrho)=s>1$ for all $\varsigma, \varrho \in M$.

Example 1. Let $M=\{0\} \cup \mathbb{N}$ and $p$ a positive even integer. Define a mapping $\eta: M \times M \rightarrow$ $[1,+\infty)$ by

$$
\eta(\varsigma, \varrho)=\left\{\begin{array}{cl}
1+|\varsigma|+|\varrho|, & \text { if } \varsigma \neq \varrho, \\
1, & \text { if } \varsigma=\varrho,
\end{array}\right.
$$

for all $\varsigma, \varrho \in M$. Define $\sigma_{b}(\varsigma, \varrho): M \times M \rightarrow A$ by $\sigma_{b}(\varsigma, \varrho)=(\varsigma+\varrho)^{p} e^{t}$, for all $\varsigma, \varrho \in M$ and for all $t \in[0,1]$. Then $\left(M, \sigma_{b}\right)$ is an extended cone $b$-metric-like space over Banach algebra. Condition $\left(\sigma_{b} 1\right)$ and $\left(\sigma_{b} 2\right)$ are clearly satisfied. Now, we prove $\left(\sigma_{b} 3\right)$ in Definition 4 is satisfied. For this, we take $\varsigma \in M$ as arbitrary then we see that

(i) If $\varsigma=\varrho$ then $\left(\sigma_{b} 3\right)$ is clear.

(ii) If $\varsigma \neq \varrho, \varsigma=v$, then

$$
\begin{aligned}
\eta(\varsigma, \varrho)\left[\sigma_{b}(\varsigma, v)+\sigma_{b}(v, \varrho)\right](t) & =(1+|\varsigma|+|\varrho|)\left[|\varsigma+v|^{p}+|v+\varrho|^{p}\right] e^{t} \\
& \geq(1+|\varsigma|+|\varrho|)|\varsigma+\varrho|^{p} e^{t} \\
& \left.\geq|\varsigma+\varrho|^{p}\right] e^{t} \\
& =\sigma_{b}(\zeta, \varrho)(t) .
\end{aligned}
$$


(iii) If $\varsigma \neq \varrho, \varrho \neq v, v \neq \varsigma$, then

$$
\begin{aligned}
\eta(\varsigma, \varrho)\left[\sigma_{b}(\varsigma, v)+\sigma_{b}(v, \varrho)\right](t) & =(1+|\zeta|+|\varrho|)\left[|\varsigma+v|^{p}+|v+\varrho|^{p}\right] e^{t} \\
& \geq\left(\frac{1+|\zeta|+|\varrho|}{2}\right)^{p}|\varsigma+v+v+\varrho|^{p} e^{t} \\
& =\left(\frac{1+|\zeta|+|\varrho|}{2}\right)^{p}|\varsigma+2 v+\varrho|^{p} e^{t} \\
& \geq|\zeta+2 v+\varrho|^{p} e^{t} \\
& \geq|\zeta+\varrho|^{p} e^{t} \\
& =\sigma_{b}(\varsigma, \varrho)(t) .
\end{aligned}
$$

Example 2. Let $M=\{0\} \cup \mathbb{N}$ and $p$ a positive even integer. Define a mapping $\eta: M \times M \rightarrow$ $[1,+\infty)$ by

$$
\eta(\varsigma, \varrho)=\left\{\begin{array}{cl}
1+|\varsigma|+|\varrho|, & \text { if } \varsigma \neq \varrho, \\
1, & \text { if } \varsigma=\varrho,
\end{array}\right.
$$

for all $\varsigma, \varrho \in M$. Define $\sigma_{b}(\varsigma, \varrho): M \times M \rightarrow A$ by $\sigma_{b}(\varsigma, \varrho)=\left(\varsigma^{p}+\varrho^{p}\right)^{p} e^{t}$, for all $\varsigma, \varrho \in M$ and for all $t \in[0,1]$. Then, $\left(M, \sigma_{b}\right)$ is an extended cone $b$-metric-like space over Banach algebra. Condition $\left(\sigma_{b} 1\right)$ and $\left(\sigma_{b} 2\right)$ are clearly satisfied. Now, we prove $\left(\sigma_{b} 3\right)$ in Definition 4 is satisfied. For this, we take $\varsigma \in M$ as arbitrary then we see that

(i) If $\varsigma=\varrho$ then $\left(\sigma_{b} 3\right)$ is clear.

(ii) If $\varsigma \neq \varrho, \varsigma=v$, then

$$
\begin{aligned}
\eta(\varsigma, \varrho)\left[\sigma_{b}(\varsigma, v)+\sigma_{b}(v, \varrho)\right](t) & =(1+|\zeta|+|\varrho|)\left[\left|\varsigma^{p}+v^{p}\right|^{p}+\left|v^{p}+\varrho^{p}\right|^{p}\right] e^{t} \\
& \geq(1+|\zeta|+|\varrho|)\left|\varsigma^{p}+\varrho^{p}\right|^{p} e^{t} \\
& \left.\geq\left|\varsigma^{p}+\varrho^{p}\right| e^{p}\right] e^{t} \\
& =\sigma_{b}(\zeta, \varrho)(t) .
\end{aligned}
$$

(iii) If $\varsigma \neq \varrho, \varrho \neq v, v \neq \varsigma$, then

$$
\begin{aligned}
\eta(\varsigma, \varrho)\left[\sigma_{b}(\varsigma, v)+\sigma_{b}(v, \varrho)\right](t) & =(1+|\zeta|+|\varrho|)\left[\left|\varsigma^{p}+v^{p}\right|^{p}+\left|v^{p}+\varrho^{p}\right|^{p}\right] e^{t} \\
& \geq\left(\frac{1+|\zeta|+|\varrho|}{2}\right)^{p}\left|\varsigma^{p}+v^{p}+v^{p}+\varrho^{p}\right|^{p} e^{t} \\
& =\left(\frac{1+|\zeta|+|\varrho|}{2}\right)^{p}\left|\varsigma^{p}+2 v^{p}+\varrho^{p}\right|^{p} e^{t} \\
& \geq\left|\varsigma^{p}+2 v^{p}+\varrho^{p}\right|^{p} e^{t} \\
& \geq\left|\varsigma^{p}+\varrho^{p}\right|^{p} e^{t} \\
& =\sigma_{b}(\varsigma, \varrho)(t) .
\end{aligned}
$$

Lemma 1. Let $A$ be the real Banach algebra and $l \in A,\left(l \neq \theta_{A}\right)$. If $\lim _{n \rightarrow+\infty} \frac{\left\|l^{n+1}\right\|}{\left\|l^{n}\right\|}$ exists, then $\lim _{n \rightarrow+\infty} \frac{\left\|l^{n+1}\right\|}{\left\|l^{n}\right\|}=\rho(l)$.

Proof. The proof follows directly from Proposition 1 and the Stolz-Cesàro theorem (let given any sequence $\left(l_{n}\right)_{n \geq 1}$ of (strictly) positive real numbers, suppose that $\lim _{n \rightarrow+\infty} \frac{l_{n+1}}{l_{n}}$ exists (finite or infinite), then $\lim _{n \rightarrow \infty} l_{n}^{\frac{1}{n}}=\lim _{n \rightarrow \infty} \frac{l_{n+1}}{l_{n}}$.) 
Now, we prove some fixed point theorems in the setting of an extended cone $b$-metriclike space over Banach algebra. Let us define a subset of $A$ as follows:

$$
P^{\star}=\left\{l \neq\left(\theta_{A}\right) \in P: \lim _{n \rightarrow+\infty} \frac{\left\|l^{n+1}\right\|}{\left\|l^{n}\right\|} \text { exists }\right\} .
$$

\section{Topology on Extended Cone $b$-Metric-like Space over Banach Algebra}

In this section, we define topology on extended cone $b$-metric-like space over Banach algebra.

Definition 5. Let $\left(M, \sigma_{b}\right)$ be an extended cone b-metric-like space over Banach algebra $A, \varsigma \in M$ and $c>\theta$, then $\sigma_{b}$-ball with centre $s$ and radius $c>\theta$ is

$$
B_{\sigma_{b}}(\varsigma, c)=\left\{\varrho \in M:\left|\sigma_{b}(\varsigma, \varrho)-\sigma_{b}(\varsigma, \varsigma)\right|<c\right\}
$$

and put $\mathfrak{B}=\left\{B_{\sigma_{b}}(\varsigma, c): \varsigma \in M\right.$ and $\left.\theta \ll c\right\}$.

Theorem 1. The collection $\mathfrak{B}=\left\{B_{\sigma_{b}}(\varsigma, c): \varsigma \in M, c>\theta\right\}$ of all open balls forms a basis for a topology $\tau_{\sigma_{b}}$ on $M$.

Proof. (i) Suppose $\varsigma \in M$. Clearly $\varsigma \in B_{\sigma_{b}}(\varsigma, c)$ for $c>\theta$. This gives $\varsigma \in B_{\sigma_{b}}(\varsigma, c) \subseteq$ $\bigcup_{\zeta \in M, c>\theta} B_{\sigma_{b}}(\varsigma, c)$.

(ii) Suppose that $a \in B_{\sigma_{b}}\left(\zeta, c_{1}\right) \cap B_{\sigma_{b}}\left(\zeta, c_{2}\right)$. Then there exists $c>\theta$ such that $B_{\sigma_{b}}(a, c) \subseteq$ $B_{\sigma_{b}}\left(\varsigma, c_{1}\right)$ and $B_{\sigma_{b}}(a, c) \subseteq B_{\sigma_{b}}\left(\varsigma, c_{2}\right)$. Suppose that $v \in B_{\sigma_{b}}(a, c)$ then $\sigma_{b}(a, v)-\sigma_{b}(a, a) \ll c$. Thus, $B_{\sigma_{b}}(a, c) \subseteq B_{\sigma_{b}}\left(\varsigma, c_{1}\right) \cap B_{\sigma_{b}}\left(\varsigma, c_{2}\right)$.

Definition 6. Let $\left(M, \sigma_{b}\right)$ be an extended cone b-metric-like space over Banach algebra. A sequence $\left\{\varsigma_{n}\right\}$ in $\left(M, \sigma_{b}\right)$ converges to a point $\varsigma \in M$ if and only if

$$
\lim _{n \rightarrow \infty} \sigma_{b}\left(\varsigma_{n}, \varsigma\right)=\sigma_{b}(\varsigma, \varsigma)
$$

Definition 7. Let $\left(M, \sigma_{b}\right)$ be an extended cone b-metric-like space over Banach algebra and $\left\{\varsigma_{n}\right\}$ be a sequence in $\left(M, \sigma_{b}\right)$ is called a $\theta$-Cauchy sequence if $\sigma_{b}\left(\varsigma_{n}, \varsigma_{m}\right)$ is a c-sequence in $A$, that is, for every $c \in$ int $P$ there is a positive integer $n_{0} \in N$ such that $\sigma_{b}\left(\varsigma_{n}, \varsigma_{m}\right) \ll c$ for all $n, m>n_{0}$.

Definition 8. Let $\left(M, \sigma_{b}\right)$ be an extended cone b-metric-like space over Banach algebra $A$ then $\left(M, \sigma_{b}\right)$ is said to be $\theta$-complete if every $\theta$-Cauchy sequence $\left\{\varsigma_{n}\right\}$ in $M$ converges to a point $\varsigma \in M$, that is,

$$
\lim _{n, m \rightarrow \infty} \sigma_{b}\left(\varsigma_{n}, \varsigma_{m}\right)=\lim _{n \rightarrow \infty} \sigma_{b}\left(\varsigma_{n}, \varsigma\right)=\sigma_{b}(\varsigma, \varsigma)=\theta .
$$

Definition 9. Let $\left(M, \sigma_{b}, A\right)$ be an extended cone b-metric-like space over Banach algebra. A mapping $G:\left(M, \sigma_{b}\right) \rightarrow\left(M, \sigma_{b}\right)$ is said to be $\sigma_{b}$-orbitally continuous at a point $u \in M$, if for some $\varsigma \in M$,

$$
\lim _{i \rightarrow+\infty} G^{n_{i}} \varsigma=u \text { implies } \lim _{i \rightarrow+\infty} G^{n_{i+1}} \varsigma=G u,
$$

if $G$ is $\sigma_{b}$-orbitally continuous at each point of $M$, then we say that $G$ is $\sigma_{b}$-orbitally continuous in $M$.

\section{Generalized Lipschitz Mappings}

In this section, we define generalized Lipschitz maps on extended cone $b$-metric-like space over Banach algebra. 
Definition 10. Let $\left(M, \sigma_{b}\right)$ be an extended cone b-metric-like space over Banach algebra. A mapping $T: M \times M$ is called a generalized Lipschitz mapping if there exists a vector $k \in P$ with $\rho(k)<1$ and for all $\varsigma, \varrho \in M$, we have

$$
\sigma_{b}(T \zeta, T \varrho) \preceq k \sigma_{b}(\varsigma, \varrho) .
$$

Example 3. Let $A, P, M$ be as in Example 2. Define a mapping $\sigma_{b}: M \times M \rightarrow A$ by

$$
\sigma_{b}(\zeta, \varrho)(t)=(\varsigma+\varrho)^{2} e^{t},
$$

for all $\varsigma, \varrho \in M$. Then $\left(M, \sigma_{b}\right)$ is an extended cone b-metric-like space over Banach algebra $A$ with $\eta(\varsigma, \varrho)=2^{p-1}$. Take $T: M \times M$ by $T \varsigma=\frac{\varsigma}{2}$, for all $\varsigma \in M$, we have:

$$
\begin{aligned}
\sigma_{b}(T \varsigma, T \varrho)(t) & =(T \varsigma+T \varrho)^{2} e^{t} \\
& =\left(\frac{\varsigma}{2}+\frac{\varrho}{2}\right)^{2} e^{t} \\
& \preceq \frac{1}{4}(\varsigma+\varrho)^{2} e^{t}
\end{aligned}
$$

where $\rho(k)<1$. Obviously, $T$ is a generalized Lipschitz map.

Now, we review some facts on $c$-sequence theory.

Definition 11 ([16]). Let $P$ be a solid cone in a Banach space E. A sequence $\left\{\varsigma_{n}\right\} \subset P$ is said to be a $c$-sequence if for each $c \gg \theta$ there exists a natural number $N$ such that $\varsigma_{n} \ll c$ for all $n>N$.

Lemma 2 ([15]). Let $P$ be a solid cone in a Banach algebra $A$. Suppose that $l \in P$ is an arbitrary vector and $\left\{\zeta_{n}\right\}$ is a c-sequence in $P$. Then, $\left\{l_{\zeta_{n}}\right\}$ is a c-sequence.

Lemma 3 ([14]). Let $A$ be a Banach algebra with a unit $e, l \in A$, then $\lim _{n \rightarrow+\infty}\left\|l^{n}\right\| \frac{1}{n}$ exists and the spectral radius $\rho(l)$ satisfies

$$
\rho(l)=\lim _{n \rightarrow+\infty}\left\|l^{n}\right\|^{\frac{1}{n}}=\inf \left\|l^{n}\right\|^{\frac{1}{n}} .
$$

If $\rho(l)<|\lambda|$, then $(\lambda e-l)$ is invertible in $A$, moreover,

$$
(\lambda e-l)^{-1}=\sum_{i=0}^{+\infty} \frac{l^{i}}{\lambda^{i+1}}
$$

where $\lambda$ is a complex constant.

Lemma 4 ([14]). Let $A$ be a Banach algebra with a unit $e, \alpha, \beta \in A$. If $\alpha$ commutes with $\beta$, then

$$
\rho(\alpha+\beta) \preceq \rho(\alpha)+\rho(\beta), \rho(\alpha \beta) \preceq \rho(\alpha) \rho(\beta) .
$$

Lemma 5 ([17]). Let $A$ be Banach algebra with a unit $e$ and $P$ be a solid cone in $A$. Let $\alpha, k, l \in P$ hold $l \preceq k$ and $\alpha \preceq$ l $\alpha$. If $\rho(k)<1$, then $\alpha=\theta$.

Lemma 6 ([17]). If $E$ is a real Banach space with a solid cone $P$ and $\left\{\varsigma_{n}\right\} \subset P$ is a sequence with $\left\|\varsigma_{n}\right\| \rightarrow 0(n \rightarrow+\infty)$, then $\left\{\varsigma_{n}\right\}$ is a c-sequence.

Lemma 7 ([17]). Let $E$ be a real Banach space with a solid cone $P$.

(1) If $\alpha, \beta, \gamma \in E$ and $\alpha \preceq \beta \ll \gamma$, then $\alpha \ll \gamma$.

(2) If $\alpha \in P$ and $\alpha \ll \gamma$ for each $\gamma \gg \theta$, then $\alpha=\theta$. 
Lemma 8 ([17]). Let $A$ be a Banach algebra with a unit e and $l \in A$. If $\lambda$ is a complex constant and $\rho(l)<|\lambda|$, then

$$
\rho\left((e-l)^{-1}\right) \preceq \frac{1}{|\lambda|-\rho(l)} .
$$

\section{Main Results}

The following theorem is our main result.

Theorem 2. Let $\left(M, \sigma_{b}, A\right)$ be a $\theta$-complete extended cone b-metric-like space over Banach algebra. Let $P$ be a solid cone in A not necessarily normal in $A$; suppose that $T: M \rightarrow M$ is a mapping such that for all $\varsigma \in M$ :

$$
\sigma_{b}\left(T \zeta, T^{2} \varsigma\right) \preceq l \sigma_{b}(\varsigma, T \zeta),
$$

where $l \in P^{*}, \rho(l)<1$ with $\lim _{n, m \rightarrow+\infty} \eta\left(\varsigma_{n}, \varsigma_{m}\right)<\frac{1}{\rho(l)}$ and $\left\{\varsigma_{n}\right\}=\left\{T^{n} \varsigma_{0}\right\}$ is the Picard iterating sequence generated by $\varsigma_{0} \in M$. Then $T$ has a fixed point in $M$ provided that $T$ is $\sigma_{b}$-orbitally in $M$.

Proof. From the contractive condition (1), we have:

$$
\begin{aligned}
\sigma_{b}\left(\varsigma_{n}, \varsigma_{n+1}\right) & =\sigma_{b}\left(T \varsigma_{n-1}, T^{2} \varsigma_{n-1}\right) \\
& \preceq l \sigma_{b}\left(\varsigma_{n-1}, T \varsigma_{n-1}\right) \\
& =l \sigma_{b}\left(\varsigma_{n-1}, \varsigma_{n}\right) \\
& \vdots \\
& \preceq l^{n} \sigma_{b}\left(\varsigma_{0}, \varsigma_{1}\right) .
\end{aligned}
$$

Now, for all $n \in N$ and for any $p=1,2, \ldots$

$$
\begin{aligned}
& \sigma_{b}\left(\varsigma_{n}, \varsigma_{n+p}\right) \preceq \eta\left(\varsigma_{n}, \varsigma_{n+p}\right)\left[\sigma_{b}\left(\varsigma_{n}, \varsigma_{n+1}\right)+\sigma_{b}\left(\varsigma_{n+1}, \varsigma_{n+p}\right)\right] \\
& =\eta\left(\varsigma_{n}, \varsigma_{n+p}\right) \sigma_{b}\left(\varsigma_{n}, \varsigma_{n+1}\right)+\eta\left(\varsigma_{n}, \varsigma_{n+p}\right) \sigma_{b}\left(\varsigma_{n+1}, \varsigma_{n+p}\right) \\
& \preceq \eta\left(\varsigma_{n}, \varsigma_{n+p}\right) \sigma_{b}\left(\varsigma_{n}, \varsigma_{n+1}\right)+\eta\left(\varsigma_{n}, \varsigma_{n+p}\right) \eta\left(\varsigma_{n+1}, \varsigma_{n+p}\right) \\
& {\left[\sigma_{b}\left(\varsigma_{n+1}, \varsigma_{n+2}\right)+\sigma_{b}\left(\varsigma_{n+2}, \varsigma_{n+p}\right)\right]} \\
& =\eta\left(\varsigma_{n}, \varsigma_{n+p}\right) \sigma_{b}\left(\varsigma_{n}, \varsigma_{n+1}\right)+\eta\left(\varsigma_{n}, \varsigma_{n+p}\right) \eta\left(\varsigma_{n+1}, \varsigma_{n+p}\right) \sigma_{b}\left(\varsigma_{n+1}, \varsigma_{n+2}\right) \\
& +\eta\left(\varsigma_{n}, \varsigma_{n+p}\right) \eta\left(\varsigma_{n+1}, \varsigma_{n+p}\right) \sigma_{b}\left(\varsigma_{n+2}, \varsigma_{n+p}\right) \\
& \vdots \\
& \preceq \eta\left(\varsigma_{n}, \varsigma_{n+p}\right) \sigma_{b}\left(\varsigma_{n}, \varsigma_{n+1}\right)+\eta\left(\varsigma_{n}, \varsigma_{n+p}\right) \eta\left(\varsigma_{n+1}, \varsigma_{n+p}\right) \sigma_{b}\left(\varsigma_{n+1}, \varsigma_{n+2}\right) \\
& +\cdots+\eta\left(\varsigma_{n}, \varsigma_{n+p}\right) \sigma_{b}\left(\varsigma_{n+1}, \varsigma_{n+p}\right) \cdots \eta\left(\varsigma_{n+p-2}, \varsigma_{n+p}\right) \\
& {\left[\sigma_{b}\left(\varsigma_{n+p-2}, \varsigma_{n+p-1}\right)+\sigma_{b}\left(\varsigma_{n+p-1}, \varsigma_{n+p}\right)\right]} \\
& \preceq \eta\left(\varsigma_{n}, \varsigma_{n+p}\right) l^{n} \sigma_{b}\left(\varsigma_{0}, \varsigma_{1}\right)+\eta\left(\varsigma_{n}, \varsigma_{n+p}\right) \eta\left(\varsigma_{n+1}, \varsigma_{n+p}\right) l^{n+1} \sigma_{b}\left(\varsigma_{0}, \varsigma_{1}\right) \\
& +\eta\left(\varsigma_{n}, \varsigma_{n+p}\right) \eta\left(\varsigma_{n+1}, \varsigma_{n+p}\right) \cdots \eta\left(\varsigma_{n+p-1}, \varsigma_{n+p}\right) l^{n+p-1} \sigma_{b}\left(\varsigma_{0}, \varsigma_{1}\right) \\
& \preceq\left[\sum_{r=0}^{n+p-1} l^{r} \prod_{s=1}^{r} \eta\left(\varsigma_{s}, \varsigma_{n+p}\right)\right] \sigma_{b}\left(\varsigma_{0}, \varsigma_{1}\right) .
\end{aligned}
$$

Again, we have:

$$
\left\|\sum_{r=n}^{n+p-1} l^{r} \prod_{s=1}^{r} \eta\left(\varsigma_{s}, \varsigma_{n+p}\right)\right\| \leq \sum_{r=n}^{n+p-1} \prod_{s=1}^{r} \eta\left(\varsigma_{s}, \varsigma_{n+p}\right) .\left\|l^{r}\right\|
$$


For $r \in N$, let us define:

$$
u_{r}^{(n+p)}=\prod_{s=1}^{r} \eta\left(\varsigma_{s}, \varsigma_{n+p}\right)\left\|l^{r}\right\|
$$

Then, for any $p=1,2,3, \ldots$

$$
\begin{aligned}
\lim _{n \rightarrow+\infty} \frac{u_{n+1}^{(n+p)}}{u_{n}^{(n+p)}} & =\lim _{n \rightarrow+\infty} \eta\left(\varsigma_{n+1}, \varsigma_{n+p}\right) \frac{\left\|l^{n+1}\right\|}{\left\|l^{n}\right\|} \\
& =\lim _{n \rightarrow+\infty} \eta\left(\varsigma_{n+1}, \varsigma_{n+p}\right) \lim _{n \rightarrow+\infty} \frac{\left\|l^{n+1}\right\|}{\left\|l^{n}\right\|} .
\end{aligned}
$$

Since $l \in P^{*}$, we have $\lim _{n \rightarrow+\infty} \frac{\left\|l^{n+1}\right\|}{\left\|l^{n}\right\|}=\rho(l)$ and, therefore, for any $p=1,2, \ldots$

$$
\lim _{n \rightarrow+\infty} \frac{u_{n+1}^{(n+p)}}{u_{n}^{(n+p)}}=\lim _{n \rightarrow+\infty} \eta\left(\varsigma_{n+1}, \varsigma_{n+p}\right) \rho(l)<1 .
$$

So, by ratio test, we have for any $p=1,2, \ldots$

$$
\lim _{n \rightarrow+\infty}\left\|\sum_{r=n}^{n+p-1} l^{n} \prod_{s=1}^{r} \eta\left(\varsigma_{s}, \varsigma_{n+p}\right)\right\| \leq \lim _{n \rightarrow+\infty} \sum_{r=n}^{n+p-1} \prod_{s=1}^{r} \eta\left(\varsigma_{s}, \varsigma_{n+p}\right)\left\|l^{r}\right\| .
$$

Therefore from (3), we conclude that $\left\{\zeta_{n}\right\}$ is Cauchy sequence in $M$. Since $M$ is complete, there exists an element $\varsigma \in M$ such that

$$
\lim _{n, m \rightarrow+\infty} \sigma_{b}\left(\varsigma_{n}, \varsigma_{m}\right)=\lim _{n \rightarrow+\infty} \sigma_{b}\left(\varsigma_{n}, \varsigma\right)=\sigma_{b}(\varsigma, \varsigma)=\theta
$$

Now, if $T$ is orbitally continuous in $M$, then $\varsigma_{0}^{n}=\varsigma_{n} \rightarrow \varsigma$ implies $T \zeta_{0}^{n}=T \varsigma_{n}=$ $\varsigma_{n+1} \rightarrow T \varsigma$. Hence $T \varsigma=\varsigma$ and $\varsigma$ is a fixed point in $M$.

From Theorem 2, we obtain the following result for generalized Lipschitz mapping in extended cone $b$-metric-like space over Banach algebra, that is, Banach theorem on a fixed point (see [18]).

Corollary 1. Let $\left(M, \sigma_{b}, A\right)$ be $\theta$-complete extended cone b-metric-like space over Banach algebra. Let $P$ be a solid cone in A not necessarily normal in A suppose that $T: M \rightarrow M$ be a mapping such that for all $\varsigma, \varrho \in M$

$$
\sigma_{b}(T \zeta, T \varrho) \preceq k \sigma_{b}(\varsigma, \varrho),
$$

where $k \in P, \rho(k)<1$ with $\lim _{n, m \rightarrow+\infty} \eta\left(\varsigma_{n}, \varsigma_{m}\right)<\frac{1}{\rho(k)}$ and $\left\{\varsigma_{n}\right\}=\left\{T^{n} \varsigma_{0}\right\}$ is the Picard iterating sequence generated by $\varsigma_{0} \in M$. Then $T$ has a fixed point in $M$ provided that $T$ is $\sigma_{b}$-orbitally in $M$.

Proof. From condition (4), if put $\varrho=T \varsigma$ we obtain condition (1).

Remark 5. Note that from condition (4) we conclude that the fixed point is unique.

Example 4. Let us consider the Banach algebra $A=C_{R}^{1}[0,1]$ with the norm $\|\varsigma\|_{A}=\|\varsigma\|_{\infty}+\|$ $\varsigma^{\prime} \|_{\infty}$ and usual pointwise multiplication obviously, $A$ is a Banach algebra with unity $e_{A}=1$. Let us take $P=\{\varsigma \in A: \varsigma(t) \geq 0$ for all $t \in[0,1]\}$. Then it can be verified that $p$ is $a$ non-normal cone. Now, let $M=\{0,1,2\}$. Define $\eta: M \times M \rightarrow[1, \infty)$ and $\sigma_{b}: M \times M \rightarrow A$ as $\eta(\varsigma, \varrho)=1+\varsigma+\varrho$, for all $\varsigma, \varrho \in M$ and $\sigma_{b}(0,0)(t)=\sigma_{b}(1,1)(t)=\sigma_{b}(2,2)(t)=\theta$ $\sigma_{b}(0,1)(t)=\sigma_{b}(1,0)(t)=e^{t}, \sigma_{b}(1,2)(t)=\sigma_{b}(2,1)(t)=4 e^{t}, \sigma_{b}(0,2)(t)=\sigma_{b}(2,0)(t)=8 e^{t}$, for all $t \in[0,1]$. Then $\left(M, \sigma_{b}\right)$ is a $\theta$-complete extended cone b-metric-like space over Banach 
algebra but not a cone metric-like space over Banach algebra. Let $T: M \rightarrow M$ be defined by $T 0=T 1=1, T 2=0$. Then, $\sigma_{b}\left(T \varsigma, T^{2} \varsigma\right) \preceq l \sigma_{b}(\varsigma, T \varsigma)$, for all $\varsigma \in M$, with $l(t)=\frac{1}{4}+\frac{t}{12}$ for all $t \in[0,1]$. We show that $l \in P^{\star}$. Then $l^{n}(t)=\left(\frac{1}{4}+\frac{t}{12}\right)^{n}$, for all $t \in[0,1]$ and for all $n \in N$. Therefore $\left(l^{n}\right)^{\prime}(t)=\frac{n}{12}\left(\frac{1}{4}+\frac{t}{12}\right)^{n-1}$ for all $t \in[0,1]$ and for all $n \in N$. Thus, $\left\|l^{n}\right\|_{A}=\left(\frac{1}{4}+\frac{t}{12}\right)^{n-1}\left[\left(\frac{1}{4}+\frac{t}{12}\right)+\frac{n}{12}\right]$ for all $n \geq 1$. Hence, we get

$$
\begin{aligned}
\frac{\left\|l^{n+1}\right\|_{A}}{\left\|l^{n}\right\|_{A}} & =\frac{\left(\frac{1}{4}+\frac{t}{12}\right)^{n}\left[\left(\frac{1}{4}+\frac{t}{12}\right)+\frac{n+1}{12}\right]}{\left(\frac{1}{4}+\frac{t}{12}\right)^{n-1}\left[\left(\frac{1}{4}+\frac{t}{12}\right)+\frac{n}{12}\right]} \\
& =\left(\frac{1}{4}+\frac{t}{12}\right)\left[\frac{t+n+4}{t+n+3}\right] \text { for all } n \geq 1
\end{aligned}
$$

So, from Equation (5) it follows that $l \in P^{\star}$. One can also check that $T$ satisfies all the conditions of Theorem 2 and $l \in M$ is the fixed point of $T$.

\section{An Application to an Integral Equation}

In this section, we endeavor to apply Corollary 1 to investigate the existence of the Fredholm integral equation.

Consider $C[0,1]$, the class of continuous functions on $[0,1]$. Let $A=C[0,1]$ be equipped with the norm $\|\varsigma\|=\|\varsigma\|_{\infty}+\left\|\zeta^{\prime}\right\|_{\infty}$. Take the usual multiplication, then $A$ is a Banach algebra with the unit $e=1$. Define $\sigma_{b}: M \times M \rightarrow A$ be the extended cone $b$-metric-like space over Banach algebra by

$$
\sigma_{b}(\zeta, \varrho)(t)=\sup _{t \in[a, b]}\left|\frac{\zeta(t)+\varrho(t)}{2}\right|^{p},
$$

where $p>1$, with $\eta(\varsigma, \varrho)=1+|\varsigma|+|\varrho|$, for all $\varsigma, \varrho \in C[0,1]$. Then $\left(\mathrm{M}, \sigma_{b}\right)$ is a $\theta$-complete extended cone $b$-metric-like space over Banach algebra.

Theorem 3. Assume that for all $\varsigma, \varrho \in C[0,1]$,

$$
\left|k\left(t, r, T_{\zeta}(t)\right)+k\left(t, r, T^{2} \zeta(t)\right)\right|^{p} \leq(1+|\zeta|+|\varrho|)^{\frac{1}{p}}|\zeta(t)+\varrho(t)|,
$$

for all $t, r \in[0,1]$. Then the integral equation:

$$
\varsigma(t)=\int_{0}^{T} k(t, r, \varsigma(t)) d r,
$$

where $t \in[0,1]$, admits a solution in $C[0,1]$.

Proof. Define $T: M \rightarrow M$ by:

$$
T \varsigma(t)=\int_{0}^{1} k(t, r, \varsigma(r)) d r, \text { for all } t, s \in[0,1]
$$


we have

$$
\begin{aligned}
\sigma_{b}\left(T \varsigma, T^{2} \zeta\right)(t) & =\sup _{t \in[0,1]}\left|\frac{T \zeta(t)+T \varrho(t)}{2}\right|^{p} e^{t} \\
& =\left|\int_{0}^{1} \frac{k(t, r, \zeta(t))+k(t, r, \varrho(t))}{2} d r\right|^{p} e^{t} \\
& \leq\left(\int_{0}^{1}\left|\frac{k(t, r, \zeta(t))+k(t, r, \varrho(t))}{2}\right| d r\right)^{p} e^{t} \\
& \leq\left(\int_{0}^{1}\left|\frac{(1+|\zeta|+|\varrho|)^{\frac{1}{p}}|\zeta(t)+\varrho(t)|}{2}\right| d r\right)^{p} e^{t} \\
& \leq \eta(\zeta, \varrho) \sigma_{b}(\zeta, \varrho)(t) .
\end{aligned}
$$

Thus, condition (1) is satisfied; therefore, all conditions of Corollary 1 are satisfied. Hence $T$ has a fixed point, which means that the Fredholm integral Equation (6) has a solution. This completes the proof.

In the end, we give some open problems.

Problem 1. Whether condition $\lim _{n, m \rightarrow+\infty} \eta\left(\varsigma_{n}, \varsigma_{m}\right)<\frac{1}{\rho(l)}$ in Theorem 2, can be replaced with condition $\lim _{n, m \rightarrow+\infty} \eta\left(\varsigma_{n}, \varsigma_{m}\right)<+\infty$ ?

Problem 2. Prove analogue results for Kannan, Chatterjee, Reich, Ćirić and Hardy-Rogers type contractions in extended cone b-metric-like space over Banach algebra.

Remark 6. Regarding Problem 2, see papers [19-23].

\section{Conclusions}

Fixed point theory is a very important tool for solving problems emerging in various domains of analysis and other fields of science. In this note, we introduce the notion of extended cone b-metric like spaces over Banach algebra where we generalized the constant $s \geq 1$ by a function $\eta(\varsigma, \varrho)$ in triangle inequality and investigated the existence and uniqueness of a fixed point using generalized Lipschitz maps. At last, some open problems are given for the readers.

Author Contributions: Investigation, J.F., N.M., A.S., M.P., and Z.D.M.; Methodology, J. F., N.M. and Z.D.M.; Software, J.F., N.M. and Z.D.M.; Supervision, J.F., N.M. and Z.D.M. All authors have read and agreed to the published version of the manuscript.

Funding: This research received no external funding.

Institutional Review Board Statement: Not applicable.

Informed Consent Statement: Not applicable.

Data Availability Statement: Not applicable.

Conflicts of Interest: The authors declare no conflict of interest.

\section{References}

1. Fréchet, M. Sur quelques points du calcul fonctionnel. Palermo Rend. 1906, 22, 1-74. [CrossRef]

2. Huang, L.G.; Zhang, X. Cone metric spaces and fixed point theorems for contractive mappings. J. Math. Anal. Appl. 2007, 332, 1468-1476. [CrossRef]

3. Liu, H.; Xu, S. Cone metric spaces with Banach algebras and fixed point theorems of generalized Lipschitz mappings. Fixed Point Theory Appl. 2013, 2013, 320. [CrossRef]

4. Fernandez, J.; Malviya, N.; Shukla, S. Cone $b$-metric-like spaces over Banach algebra and fixed point theorems with application. Asian J. Math. Comp. Res. 2017, 18, 49-66. 
5. Fernandez, J.; Malviya, N.; Djekic-Dolićanin, D.; Pučić, D. The $p_{b}$-cone metric spaces over Banach algebra with applications. Filomat 2020, 34, 983-998. [CrossRef]

6. Fernandez J.; Saelee S.; Saxena K.; Malviya N.; Kumam P. The A-cone metric space over Banach algebra with application of generalized Lipschitz and expansive maps in fixed point theory and integral equations. Cogent Math. 2017, 3, 1282690. [CrossRef]

7. Mitrović, Z.D.; Hussain, N. On results of Hardy-Rogers and Reich in cone b-metric space over Banach algebra and applications. UPB Sci. Bull. Ser. A 2019, 81, 147-154.

8. Mitrović, Z.D.; Aydi, H.; Radenović, S. On Banach and Kannan type results in cone bv(s)-metric spaces over Banach algebra. Acta Math. Univ. Comen. 2020, 1, 143-150.

9. Mitrović, Z.D.; Ahmed, A.; Salunke, J.N. A cone generalized b-metric like space over Banach algebra and contraction principle. Thai J. Math. 2021, 19, 583-592.

10. Roy, K.; Panja, S.; Saha, M. A Generalized Fixed Point Theorem in an Extended Cone $b$-Metric Space Over Banach Algebra with Its Application To Dynamical Programming. Appl. Math. E-Notes 2021, 21, 209-219.

11. Shatanawi, W.; Mitrović, Z.D.; Hussain, N.; Radenović, S. On Generalized Hardy-Rogers Type $\alpha$-Admissible Mappings in Cone b-Metric Spaces over Banach Algebras. Symmetry 2020, 12, 81. [CrossRef]

12. Radenović, S.; Rhoades, B.E. Fixed point theorem for two non-self mappings in cone metric spaces. Comput. Math. Appl. 2009, 57, 1701-1707. [CrossRef]

13. Rezapour, S.; Hamlbarani, R. Some notes on the paper, "Cone metric spaces and fixed point theorems of contractive mappings". $J$. Math. Anal. Appl. 2008, 345, 719-724. [CrossRef]

14. Rudin, W. Functional Analysis, 2nd ed.; McGraw-Hill: New York, NY, USA, 1991.

15. Xu, S.; Radenović, S. Fixed point theorems of generalized Lipschitz mappings on cone metric spaces over Banach algebras without assumption of normality. Fixed Point Theory Appl. 2014, 2014, 102. [CrossRef]

16. Kadelburg, Z.; Radenović, S. A note on various types of cones and fixed point results in cone metric spaces. Asian J. Math. Appl. 2013, 2013, 1-7.

17. Huang, H.; Radenović, S. Common fixed point theorems of Generalized Lipschitz mappings in cone metric spaces over Banach algebras. Appl. Math. Inf. Sci. 2015, 9, 2983-2990. [CrossRef]

18. Banach, S. Sur les opérations dans les ensembles abstraits et leur application aux équations intégrales. Fund. Math. 1922, 3, 133-181. [CrossRef]

19. Chatterjee, S.K. Fixed point theorem. Comptes Rend. Acad. Bulgaria Sci. 1972, 25, 727-730. [CrossRef]

20. Ćirić, L.B. A generalization of Banach's contraction principle. Proc. Am. Math. Soc. 1974, 45, 267-273. [CrossRef]

21. Kannan, R. Some results on fixed point. Bull. Cal. Math. Soc. 1958, 60, 71-76.

22. Reich, S. Some remarks concerning contraction mappings. Can. Math. Bull. 1971, 14, 121-124. [CrossRef]

23. Rhoades, B.E. A comparison of various definitions of contractive mappings. Trans. Am. Math. Soc. 1977, 226, 256-290. [CrossRef] 